Research Article

\title{
Effect of Arm Position on Image Quality and Radiation Dose in Multidetector Computed Tomography
}

\author{
Dae Cheol Kweon ${ }^{1}$ and Jiwon Choi ${ }^{2, *}$ \\ ${ }^{1}$ Department of Radiological Science, Shinhan University, Uijeongbu, South Korea \\ ${ }^{2}$ Department of Radiological Science, Jeonju University, Jeonju, South Korea \\ "Corresponding author: Department of Radiological Science, Jeonju University, 303, Cheonjam-ro, Wansan-gu, 55069 Jeonju, Jeollabuk-do, South Korea. Tel: +82-632203260, Fax: \\ 82-632202054,Email: jwchoi@jj.ac.kr
}

Received 2018 November 11; Revised 2019 June 06; Accepted 2019 July 03.

\begin{abstract}
Background: Abdominal CT using a tube-current modulation technique may result in artifacts due to changes in the position of the patient's arms, resulting in poor image quality and excessive radiation exposure. To solve these problems, the patient's arm is positioned mainly on the head or outside the examination area.

Objectives: An optimization method to acquire high-quality images in CT examination by comparing the radiation dose and image according to the change in arm position and the use of the tube-current modulation method in CT examination.

Materials and Methods: To analyze the CT images, the mean, standard deviation (SD), and coefficient of variation (COV) of the Hounsfield unit (HU) of the CT were obtained by measuring the pixel values of the heart, chest, lung, and bone using region of interest (ROI) manage. The dose of computed tomography dose index (CTDI) and dose length product (DLP) according to the position of the arm.

Results: The arms up position of $\mathrm{CTDI}_{\mathrm{vol}}$ and DLP values were lower than the arms down position. In the z-axis thickness modulation (Z-DOM), the tube current increased in the shoulders, decreased in the lungs, and increased in the abdomen. There was no artifact when the arms were raised, but an artifact was present when the arm was lowered. The calculated peak signal-to-noise ratio (PSNR) for the Z-DOM application in the CT scan of the anthropomorphic phantom by applying automatic exposure control (AEC) was 38.21 $\mathrm{dB}$. There was no significant difference between SD and COV using Z-DOM and the fixed tube current technique $(\mathrm{P}>0.05)$. Noise in the image increased when the arm was raised, and dose increased in the thyroid and upper chest.

Conclusion: The position of the upper arms directly affects the image in the MDCT imaging of the anthropomorphic phantom. Therefore, it is possible to increase the quality of the image by reducing the amount of artifacts and the amount of radiation by orienting the arms upward. This optimal test method could be used in clinical practice to achieve high image quality and low noise in the MDCT of trauma patients.
\end{abstract}

Keywords: Anthropomorphic Phantom, CTDI, Dose Modulation, Pixel Value, MDCT

\section{Background}

Because of the rapid development of medical technology and computers, computed tomography (CT) tests play an important role in diagnostic imaging. In clinical practice, CT scanners are used for the rapid treatment of emergency patients and multiple-trauma patients. CT scans have been used to diagnose and treat unstable patients with multiple injuries (1).

Optimization efforts are needed for active radiological protection in abdominal CT examinations in which organs with a high-radiation tissue-weighting factor(WT) are gathered to reduce radiation exposure. Optimization tech- niques include tube-current modulation techniques using selected reference images, tube-current modulation along the z-axis of a patient, or tube-current modulation according to an angle due to elliptical cross-section of the human body. Abdominal CT has been applied in clinical practice through tube-current modulation techniques. However, abdominal CT using a tube-current modulation technique may result in artifacts due to changes in the position of the patient's arms, resulting in poor image quality and excessive radiation exposure. To solve these problems, the patient's arm is positioned mainly on the head or outside the examination area. However, when the position of the patient's arm is not in the normal position, the problem of de- 
terioration in image quality arises and it is difficult to diagnose the artifact due to the linear hardening phenomenon and photon shortage in the CT image.

Analysis of the existing quantitative images for CT image evaluation involves the evaluation of the image using noise, contrast noise ratio (CNR), signal-to-noise ratio (SNR ), and coefficient of variation (COV) of the image $(2,3)$. In the analysis of CT images according to the position of the arm, images were quantitatively analyzed by using noise, CNR, and SNR (4). In an emergency CT scan, trauma patients cannot lift their arms over their heads due to trauma injuries. The position under the arm has been reported to reduce the diagnostic value of artifacts due to hardening of the spine in the liver, spleen, and back of the kidney (4). In addition, the location of the upper arms with the chest has been reported to degrade image quality due to beam hardening artifacts in the liver, spleen, and kidney $(5,6)$.

\section{Objectives}

The purpose of this study is to evaluate the effect of arm position on the images in an anthropomorphic CT phantom. We propose an optimization method to acquire highquality images in CT examination by comparing the radiation dose and image according to the change in arm position and the use of the tube-current modulation method in CT examination.

\section{Materials and Methods}

\subsection{In vitro Study and RPL Measurement}

We scanned an anthropomorphic phantom (PBU-60, Kyoto Kagaku Co., Ltd., Kyoto, Japan) with a body weight of $50 \mathrm{~kg}$ and a height of $165 \mathrm{~cm}$ by using a CT system (Brilliance CT 64- channel, Philips, The Netherlands) (7). The phantom was used to evaluate the radiation dose and image according to the position of the arm. The scan parameters applied to the CT scan are given in Table 1 . The following scan parameters were kept identical for all anthropomorphic phantom data acquisitions in multidetector computed tomography (MDCT) examinations: tube voltage 120 $\mathrm{kV}$; reference tube current-time product of $188 \mathrm{mAs}$, using attenuation-based tube-current modulation [z-axis thickness modulation (Z-DOM); Philips]; pitch 0.89; slice collimation $64 \mathrm{~mm} \times 0.625 \mathrm{~mm}$, gantry rotation time $0.75 \mathrm{sec}-$ onds; slice thickness, $3 \mathrm{~mm}$; reconstruction increment, 3 $\mathrm{mm}$.

The radiophotoluminescent (RPL) glass dosimetry system (ATGC, Asahi Techno Glass Corporation, Tokyo, Japan)

\begin{tabular}{|c|c|}
\hline Parameters & Description or value \\
\hline Tube voltage, $\mathbf{k V}$ & 120 \\
\hline Tube-current modulation & Z-DOM \\
\hline Slice thickness, $\mathrm{mm}$ & 3 \\
\hline Beam collimation, mm & $64 \times 0.625$ \\
\hline Image matrix (rows and columns) & $512 \times 512$ \\
\hline Pitch & 0.89 \\
\hline Field of view, mm & 350.0 \\
\hline Rotation time, $s$ & 0.75 \\
\hline Table feed, $\mathrm{mm} / \mathrm{s}$ & 47.5 \\
\hline Slice increment, mm & 3.00 \\
\hline Filter type & Y-sharp (YA) \\
\hline Convolution kernel & Y-sharp (YA) \\
\hline
\end{tabular}

Abbreviation: Z-DOM, z-axis thickness modulation

is an activated phosphate glass plate. In this measurement study, the RPL dosimeter was investigated by using a ${ }^{137} \mathrm{Cs}$ source, and the dose correction factor of the RPL was determined by comparing the RPL dose of the measured dose with the standard exposure of ${ }^{60} \mathrm{Co}$ gamma radiation. The results for energy dependence have shown that only GD352M (Element Holder: ATGC, Asahi Techno Glass Corporation, Tokyo, Japan) is suitable for use in medical exposures. The RPL dose measurement system is a Dose Ace FGD-1000 (ATGC, Asahi Techno Glass Corporation, Tokyo, Japan) used for measuring the radiation dose of DGD-1000 (ATGC, Asahi Techno Glass Corporation, Tokyo, Japan).

\subsection{Image Quality Analysis}

To analyze the CT images, digital imaging and communications in medicine (DICOM) image-processing software (ImageJ Version 1.43u; National Institutes of Health, Bethesda, MD, USA) was used to measure the various pixel values of the images. The mean Hounsfield unit (HU), standard deviation (SD) values and COV were analyzed by measuring the pixel values of anthropomorphic phantom heart, chest, lung, and bone by setting the same size region of interest (ROI) of $1 \mathrm{~cm}^{2}$ using ImageJ's ROI manage (Figure 1). The radiological technologist who carried out the research drew the ROI directly from the anthropomorphic phantom image. The examiner measured the 4 portions (heart, chest, lung, and bone) of each 3 times on each of the anthropomorphic phantom.

We calculated the SD of the HU of the CT representing the noise in the image of the anthropomorphic phantom. 

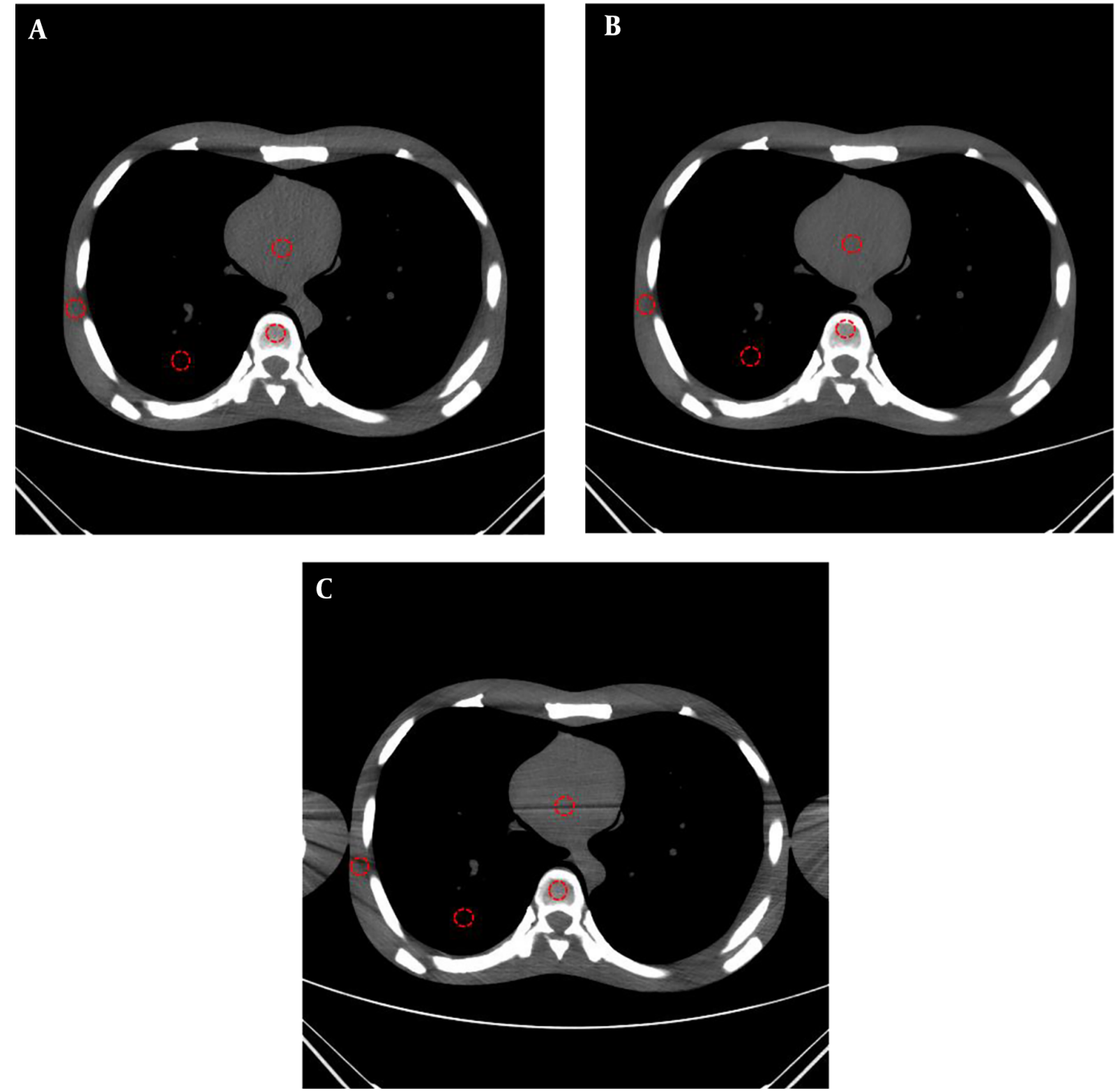

Figure 1. Examples of region of interest (ROI) position in the heart, chest, axilla, lung, and bone region. Both arms raised position (A) and z-axis thickness modulation (Z-DOM) dose modulation (B) and arms down of CT image (C).

The COV was then estimated as the ratio of $\mathrm{SD}(\sigma)$ to the mean value $(\mu)$. The mean of the CT HUs of all anthropomorphic phantoms is the SD of the measured SD $(\sigma)$ values. The COV was calculated by using Equation 1 to evaluate whether the CT image noise was more uniform when the automatic exposure control (AEC) (Z-DOM) system was deactivated by comparison.

$\operatorname{cov}=\frac{\sigma}{\mu} 100 \%$
3.3. CTDIvol (Computed Tomography Dose Index) and Radiation Dose Measurement

Volume CT dose index $\left(\mathrm{CTDI}_{\mathrm{vol}}\right)$ is an index that can measure the irradiation dose per tissue section without depending on the scan length. The dose length product (DLP), which is the total amount of irradiation dose, can be calculated through $\mathrm{CTDI}_{\mathrm{vol}}$. DLP is useful in assessing the effective dose of the entire area, which the patient is receiving. The dose information of the DICOM file was analyzed 
to evaluate the $\mathrm{CTDI}_{\mathrm{vol}}$ in the scan image of the anthropomorphic phantom. The CT dose was calculated by multiplying the DLP provided by the scanner console with the conversion factor $\left(\mathrm{k} \leq 0.017 \mathrm{mSv} \mathrm{mGy}^{-1} \mathrm{~cm}^{-1}\right)(8,9)$.

\subsection{Statistical Analysis}

Statistica analyses were performed by SPSS for windows ver. 20 (IBM Corp. Released 2011. IBM SPSS Statistics for Windows, version20.0. Armonk, NY). An unpaired Student's $t$-test was used to compare the continuous values such as $\mathrm{CTDI}_{\mathrm{vol}}$, DLP between both arms and raised in the anthropomorphic phantom. Comparisons between eye, thyroid, breast and humerus for four radiation dose datasets was performed using one-way analysis of variance (ANOVA) and compared to the 95\% confidence interval for the HU of the standardized CT, the difference was analyzed. Post analysis was performed without assuming the same distribution using the Dunnett's post-hoc test. Statistical significance was considered when the Pvalue was less than 0.05 .

\section{Results}

The noise in the anthropomorphic phantom CT image was analyzed according to the change in the position of upper arms and Z-DOM application. Standard CT scanning parameters for the abdomen were used to perform image quality measurements by evaluating the HU of the CT, SD of the noise, and COV of the available ROIs in the scanner. The dose of CTDI and DLP according to arm position increased with a CTDIvol of $7 \mathrm{mGy}$ when the arm was raised and 116 mGy when it was lowered. The DLP was $246.61 \mathrm{mGy} \cdot \mathrm{cm}$ and the effective dose was $4.4 \mathrm{mSv}$, when the arm was raised, and when the hand was down the corresponding values were $436.39 \mathrm{mGy} \cdot \mathrm{cm}$ and $7.4 \mathrm{mSv}$, respectively, higher than when the hand was raised (Table 2 ). We found a significant radiation dose between both arms raised and down $(\mathrm{P}<$ 0.05).

\begin{tabular}{lccc}
\hline Table 2. CTDI and DLP & & & \\
\hline & Both arms raised & Both arms down & P value \\
\hline CTDI $_{\text {vol }}, \mathbf{m G y}$ & 7 & 11.6 & 0.000 \\
DLP, $\mathbf{m G y} \cdot \mathbf{c m}$ & 264.61 & 436.39 & 0.000 \\
\hline
\end{tabular}

Abbreviations: $\mathrm{CTDI}_{\mathrm{vol}}$, volume CT dose index; DLP, dose length product.

The radiation dose in the CT scans using an anthropomorphic phantom with the arm raised measured by using a glass dosimeter is shown in Table 3. Radiation dose in different anatomical locations based on the arm position with thyroid gland were $16.185-22.775 \mu \mathrm{Gy}$, breast were $15.545-18.005 \mu \mathrm{Gy}$ and humerus position calculated were $862-1.428 \mu \mathrm{Gy}$.

The dose comparison when the arm is raised and lowered is shown in Figure 2. The changes in tube currents in the Z-DOM are shown in Figure 3; the tube current in the shoulder part increased, that in the lung decreased, and that in the abdomen increased.

When the anthropomorphic phantom was raised, the artifact was not present (Figure 4A), but when the upper arms were lowered, the artifact was present (Figure 4B). The peak signal-to-noise-ratio(PSNR) was measured as 13.24 $\mathrm{dB}$ in the ImageJ program with the fixed tube current technique (Figure 5A) and Z-DOM (Figure 5B) for AEC application. The PSNR in the CT scan of the anthropomorphic phantom calculated by using AEC was $38.21 \mathrm{~dB}$ (Table 4).

There was no significant difference in the mean, SD, and COV of the heart, chest, lung, and bone using the ZDOM and fixed tube current method in the anthropomorphic phantom images $(\mathrm{P}>0.05)$. However, there was a significant difference $(\mathrm{P}<0.05)$ when the arm was lowered and CT scan was performed (Table 5). Noise in the image increased when the arm was raised to a height and the dose increased in the thyroid and upper chest.

\section{Discussion}

The purpose of this study was to compare the radiological dose and image quality of the arms with the anthropomorphic phantom and glass dosimeter. Whole-body MDCT testing can provide accurate and quick results for the diagnosis and treatment of traumatic injuries. In general, additional abdominal injury can be prevented by bringing the two arms to the side of the body rather than lifting them above the head (10) before performing abdominal CT. The disadvantage of positioning the arm with the patient's torso is that the image quality of the posterior part of the liver and spleen of the abdominal organs is reduced $(6,11$, 12).

According to the results of this study, when the arm was not lifted above the head, the image quality with of the liver and spleen was significantly better than side-byside with the body when the arm was placed on the chest because there was no beam hardening artifact.

The number of patients suffering from multiple injuries is increasing, as is the importance of CT examinations $(13,14)$. A major disadvantage of CT scan is that the patient's radiation exposure is three to five times higher 


\begin{tabular}{|c|c|c|c|c|}
\hline No. & Region & Position & Both arms down & Both arms raised \\
\hline $\mathbf{1}$ & \multirow{4}{*}{ Eye } & \multirow{2}{*}{ Left } & 856 & 421 \\
\hline 2 & & & 1.022 & 538 \\
\hline 3 & & \multirow{2}{*}{ Right } & 791 & 445 \\
\hline 4 & & & 768 & 643 \\
\hline 5 & \multirow{4}{*}{ Thyroid } & \multirow{2}{*}{ Left } & 21.595 & 20.895 \\
\hline 6 & & & 24.455 & 16.185 \\
\hline 7 & & \multirow{2}{*}{ Right } & 20.855 & 18.455 \\
\hline 8 & & & 24.685 & 22.775 \\
\hline 9 & \multirow{4}{*}{ Breast } & \multirow{2}{*}{ Left } & 18.855 & 15.545 \\
\hline 10 & & & 21.045 & 17.425 \\
\hline 11 & & \multirow{2}{*}{ Right } & 19.365 & 17.555 \\
\hline 12 & & & 20.765 & 18.005 \\
\hline 13 & \multirow{4}{*}{ Humerus } & \multirow{2}{*}{ Left } & 12.405 & 862 \\
\hline 14 & & & 12.455 & 1.060 \\
\hline 15 & & \multirow{2}{*}{ Right } & 9.197 & 1.235 \\
\hline 16 & & & 10.535 & 1.428 \\
\hline
\end{tabular}

Table 4. Image Analysis of Arm Position and Dose Modulation

\begin{tabular}{|c|c|c|c|c|}
\hline & SNR & PSNR, dB & RMSE & MAE \\
\hline Both arms raised vs. both arms down & 12.59 & 13.24 & 202.85 & 71.10 \\
\hline Z-DOM vs. fixed tube current & 37.56 & 38.21 & 11.44 & 7.04 \\
\hline
\end{tabular}

Abbreviations: db, decibel; MAE: mean absolute error; PSNR, peak signal-to-noise ratio; RMSE, root mean square error; SNR, signal-to-noise ratio; Z-DOM, z-axis thickness modulation

\begin{tabular}{|c|c|c|c|c|}
\hline Parameter/position & Mean \pm SD & Minimum & Maximum & COV, \% \\
\hline \multicolumn{5}{|c|}{ Both arms raised (Z-DOM) } \\
\hline Heart & $-6.14 \pm 8.39$ & -25 & 16 & -136.64 \\
\hline Chest & $-20 \pm 9.3$ & -45 & 3 & -46.5 \\
\hline Lung & $-993.68 \pm 5.48$ & -1008 & -980 & -0.55 \\
\hline Bone & $341.92 \pm 148.91$ & 215 & 8610 & 43.55 \\
\hline \multicolumn{5}{|c|}{ Both arms raised (fixed tube current) } \\
\hline Heart & $-2.58 \pm 4.81$ & -19 & 9 & -186.43 \\
\hline Chest & $-21 \pm 9.4$ & -44 & -4 & -44.76 \\
\hline Lung & $-994.24 \pm 4.14$ & -1005 & -983 & -0.41 \\
\hline Bone & $393.98 \pm 151.17$ & 18 & 810 & 38.36 \\
\hline \multicolumn{5}{|l|}{ Both arms down } \\
\hline Heart & $-13.73 \pm 51.82$ & -132 & 55 & -377.42 \\
\hline Chest & $-13.9 \pm 22.96$ & -62 & 27 & -165.17 \\
\hline Lung & $-995.66 \pm 10.52$ & -1019 & -967 & -1.05 \\
\hline Bone & $342.5 \pm 163.87$ & -5 & 885 & 47.84 \\
\hline
\end{tabular}

Abbreviations: AEC, Automatic exposure control; COV, Coefficient of variation; SD, Standard deviation; Z-DOM, z-axis thickness modulation

than that in conventional radiology. On the other hand, systemic CT has been reported to make clinically relevant diagnosis much more frequently than general CT, and the use of whole-body CT has been shown to increase survival probability after multiple injuries (15). For these reasons, there is a continuing effort to perform CT screening faster 

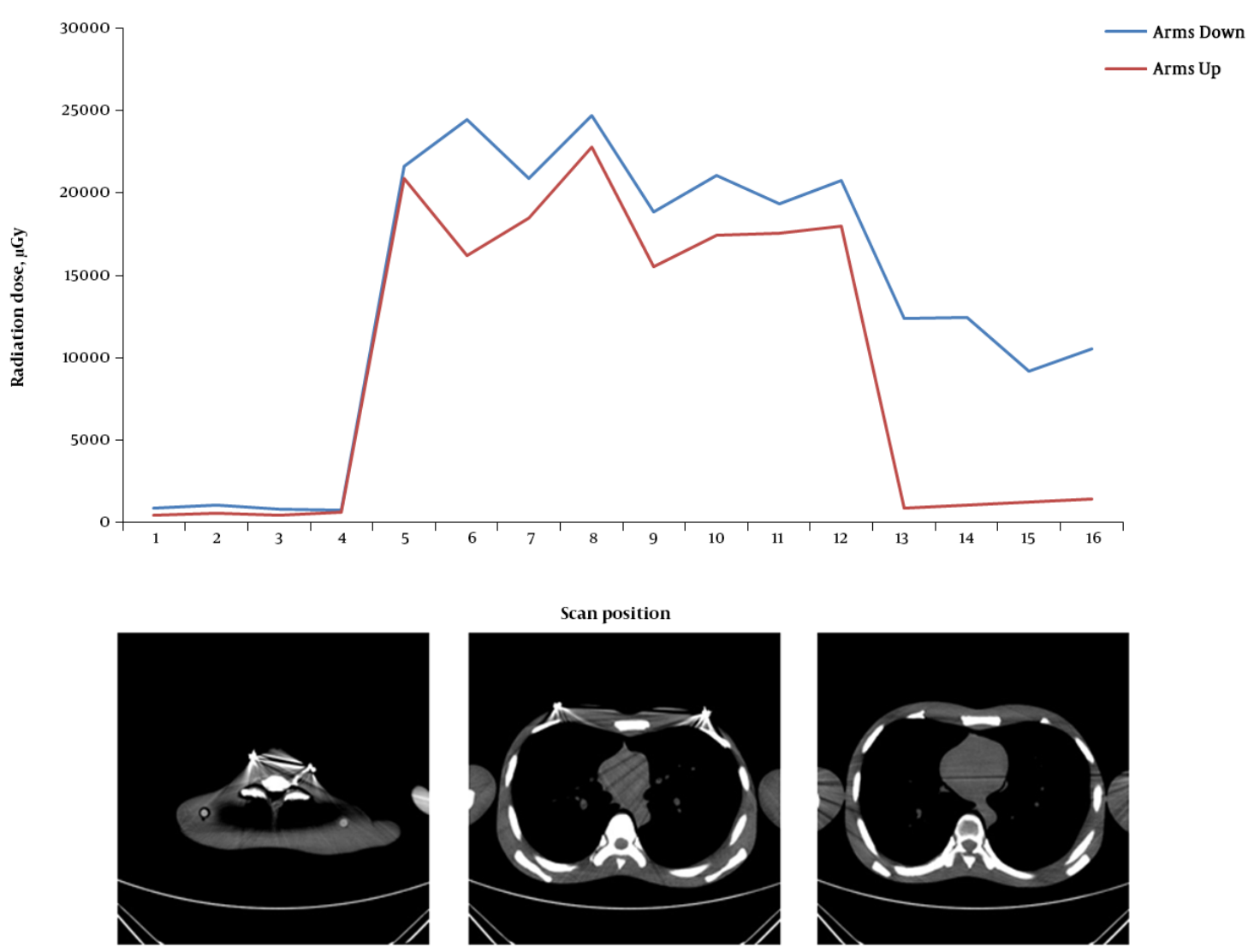

Figure 2. Radiation dose according to scan length for anthropomorphic phantom position with both arms in down and raised position

and at a lower dose.

In our study, a $\mathrm{CTDI}_{\mathrm{vol}}$ was $7 \mathrm{mGy}$ when the arm was raised and $116 \mathrm{mGy}$ when the hand was lowered. When the arm was raised DLP was $246.61 \mathrm{mGy} \cdot \mathrm{cm}$ and the effective dose was $4.4 \mathrm{mSv}$. When the hand was lowered DLP was $436.39 \mathrm{mGy} \cdot \mathrm{cm}$ and the effective dose was $7.4 \mathrm{mSv}$, which was higher than when the hands were raised.

In the study of existing effective doses, the effective dose in this study was included in $5-7 \mathrm{mSv}$ in the chest CT, and $7.4 \mathrm{mSv}$ in $8 \mathrm{mSv}$ to $15 \mathrm{mSv}$ in the abdomen. The amount of radiation was determined (16). Brink et al. (5) suggested using a tool to optimize the arm of the trauma patient in a CT scan, compared to increasing the arm above the $8 \mathrm{mSv}$ head when the arm was lowered.

In the case of using the Z-DOM in the measurement of the radiation dose with the glass dosimeter, the tube current increased in the shoulder part when the hand was raised, decreased in the lung, and increased in the abdomen. When the upper body of the anthropomorphic phantom was raised, there was no artifact, but when the upper body was lowered, an artifact occurred. This is consistent with the results of a study in which patients were placed on the upper arm and the dose was decreased and the quality of the image was higher than that of the lower position $(4,10)$. PSNR was measured as $13.24 \mathrm{~dB}$ in the fixedtube current technique and Z-DOM image comparison for AEC application. AEC was applied to the A PSNR of $38.21 \mathrm{~dB}$ was calculated for the Z-DOM application in the CT scan of the anthropomorphic phantom. This means that higher PSNR values provide better picture quality, better quality at $40 \mathrm{~dB}$, slightly lower image quality at $30 \mathrm{~dB}$, and increased noise at $20 \mathrm{~dB}$. A noise increase of $10 \mathrm{~dB}$ continues, and 0 $\mathrm{dB}$ image for strong noise intensity (17).

The Philips CT AEC system applied to the study consists of three parts. Patient-based AEC, D-DOM according to angle provision, and longitudinal Z-DOM $(18,19)$. In this study, the dose was calculated by applying Z-DOM. There was no significant difference in the mean, SD, and COV of 


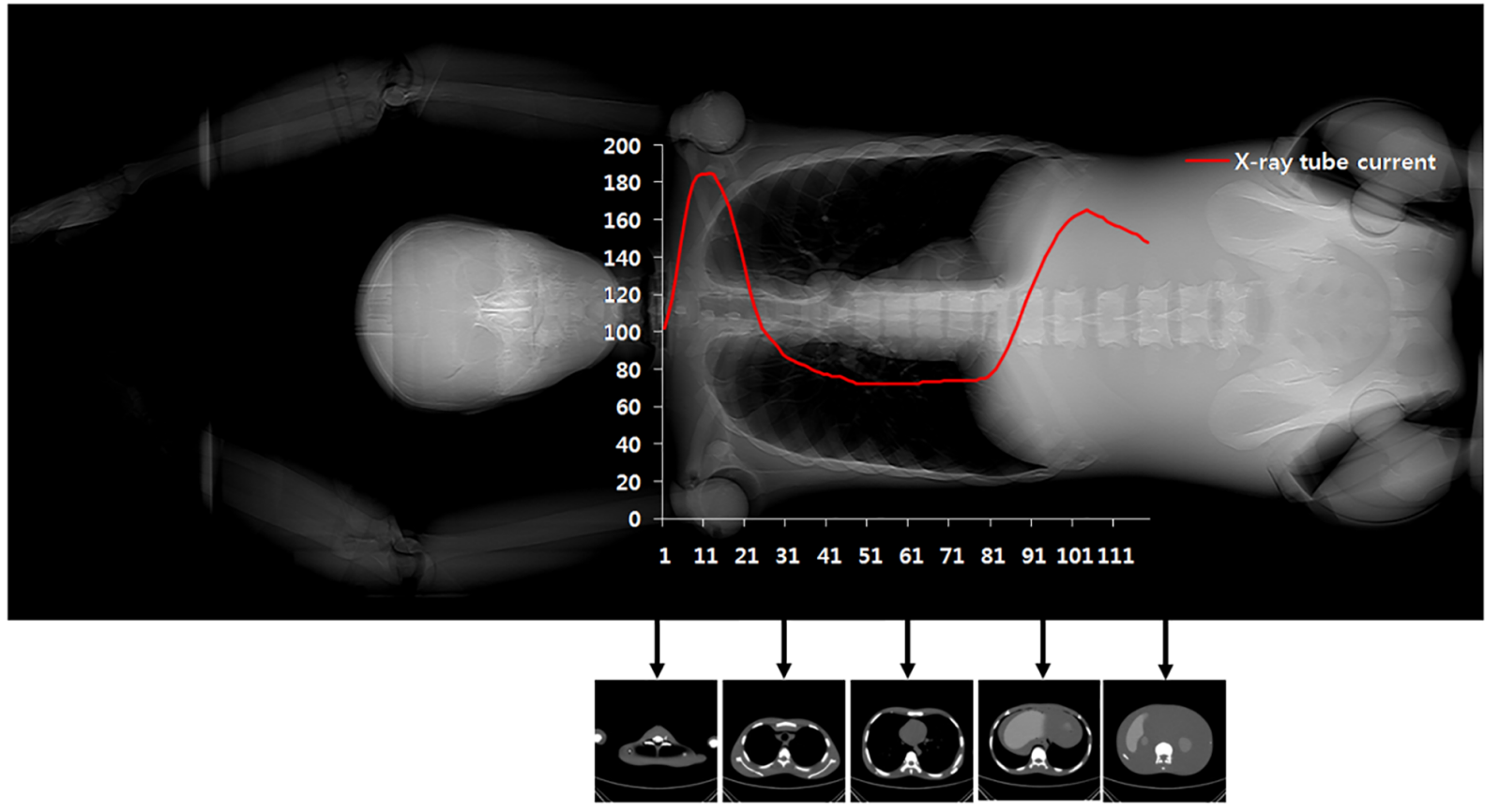

Figure 3. Radiation dose of scan length for anthropomorphic phantom position with both arms raised according to X-ray tube current
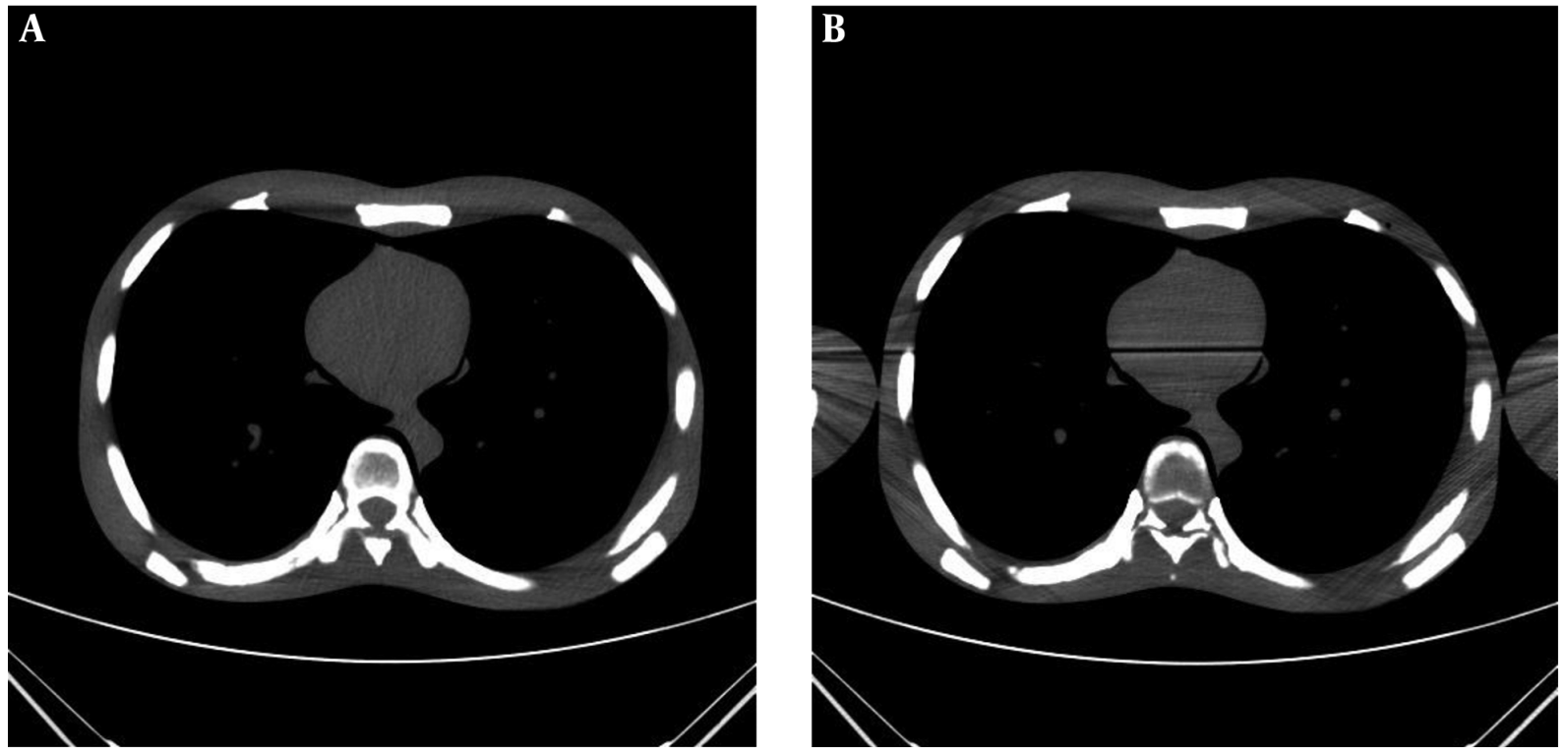

Figure 4. Both arms raised position (A) and down (B) manipulated multidetector CT scans obtained in anthropomorphic phantom imaged with both arms alongside the torso

the heart, chest, lung, and bone using Z-DOM and fixed tube current technique $(\mathrm{P}>0.05)(\mathrm{P}<0.05)$. Previous studies have shown no significant difference in imaging and dose reduction has been reported by the AEC technique
(20). When the arm was raised, the noise of the anthropomorphic phantom image increased, and the dose increased at the thyroid and chest.

Our study did not extend to the neck and lower ab- 

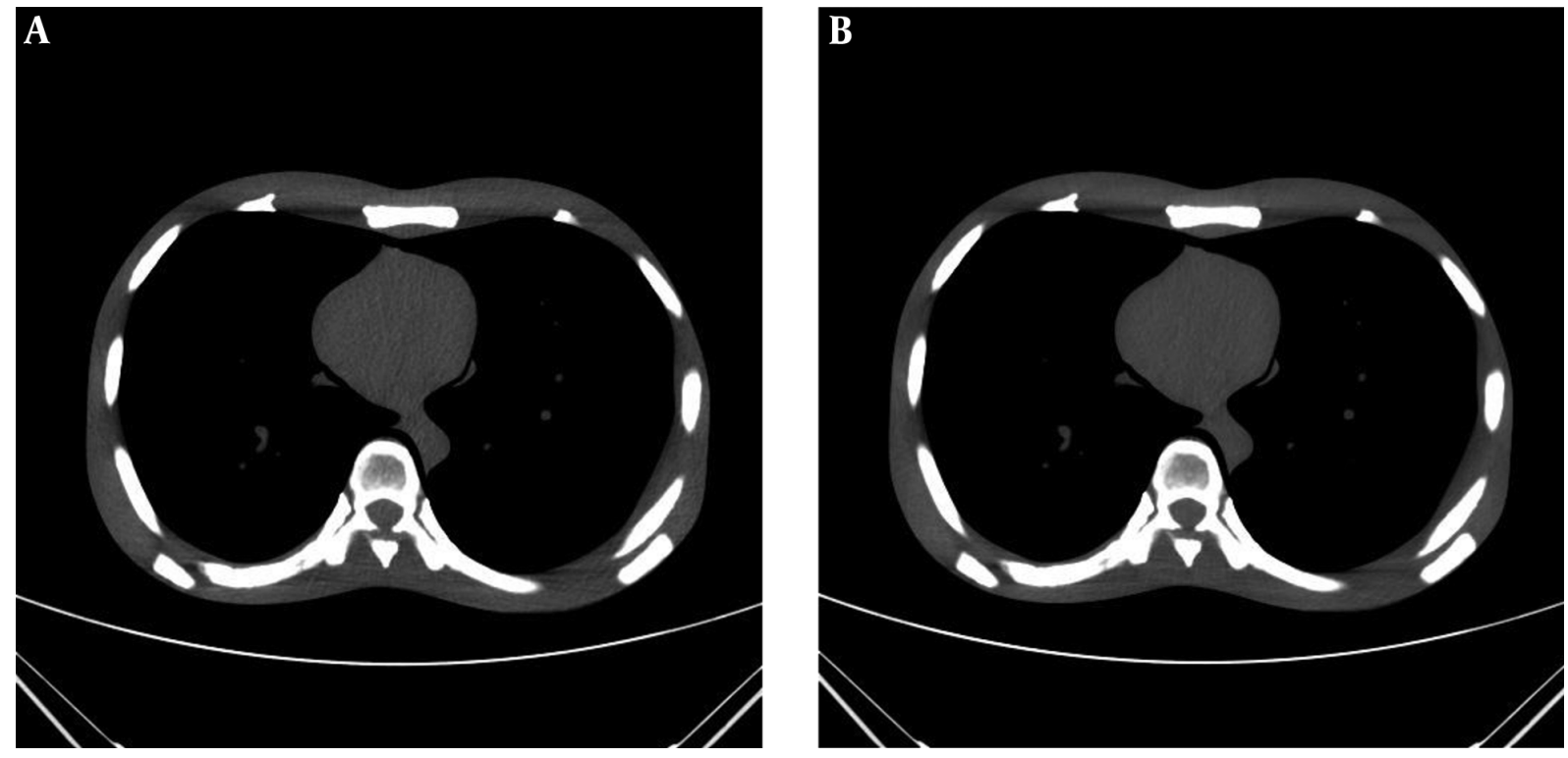

Figure 5. Original of both arms raised position (A) and z-axis thickness modulation (Z-DOM) dose modulation of CT image (B)

domen in the chest and abdominal examinations of anthropomorphic phantoms. However, there is a growing need for additional studies on the lower abdomen. In the future, there is an increasing need for studies involving clinical patients with scan range, various tube-current settings, AEC application, and trauma patients (1).

The limitations of the study are as follows: First, an anthropomorphic phantom was used and the study was not applied to real patient. Second, the AEC was applied to the anthropomorphic phantom and the arm was not scanned. Third, a variety of MDCT models and AEC applications are needed. Fourth, the quality and dose of images are measured in the lung and abdomen area. Finally, the dose was measured with a glass dosimeter. However, various measurements and further studies are needed to measure the dose of the surface without applying the internal dose.

In conclusion, the position of the upper arms directly affects the image in the MDCT imaging of the anthropomorphic phantom. Therefore, placing the position of the arm above the head can improve the image quality by reducing artifacts and radiation dose. The results of this study could be applied in clinic using this optimal method as a method to achieve high image quality and low noise in MDCT of trauma patients.

\section{Footnotes}

Authors' Contributions: Study concept and design, Dae Cheol Kweon; acquisition of data, Dae Cheol Kweon; analysis and interpretation of data, Dae Cheol Kweon, and Jiwon Choi; drafting of the manuscript, Dae Cheol Kweon; critical revision of the manuscript for important intellectual content, Jiwon Choi; statistical analysis, Dae Cheol Kweon; administrative, technical, and material support, Jiwon Choi

Conflict of Interests: The researcher claims no conflicts of interest.

Ethical Considerations: The paper, the final version of which I enclose, is not substantially the same as any that I/we have already published elsewhere

Financial Disclosure: The authors declare that they have no financial interests related to the material in the manuscript.

Funding/Support: This study was not funded by any funding source.

\section{References}

1. Nguyen D, Platon A, Shanmuganathan K, Mirvis SE, Becker CD, Poletti PA. Evaluation of a single-pass continuous whole-body 16-MDCT protocol for patients with polytrauma. AJR Am J Roentgenol. 2009;192(1):310. doi: 10.2214/AJR.07.3702. [PubMed: 19098172].

2. Tanaka C, Ueguchi T, Shimosegawa E, Sasaki N, Johkoh T, Nakamura $\mathrm{H}$, et al. Effect of CT acquisition parameters in the detection of subtle 
hypoattenuation in acute cerebral infarction: A phantom study. AJNR Am J Neuroradiol. 2006;27(1):40-5. [PubMed: 16418353].

3. Siegel MJ, Schmidt B, Bradley D, Suess C, Hildebolt C. Radiation dose and image quality in pediatric CT: Effect of technical factors and phantom size and shape. Radiology. 2004;233(2):515-22. doi: 10.1148/radiol.2332032107. [PubMed:15358847].

4. Jeon PH, Kim HJ, Lee CL, Kim DH, Lee WH, Jeon SS. Whole-body CT in polytrauma patients: The effect of arm position on abdominal image quality when using a human phantom. J Korean Phys Soc. 2012;60(11):1967-72. doi:10.3938/jkps.60.1967.

5. Brink M, de Lange F, Oostveen LJ, Dekker HM, Kool DR, Deunk J, et al. Arm raising at exposure-controlled multidetector trauma CT of thoracoabdominal region: Higher image quality, lower radiation dose. Radiology. 2008;249(2):661-70. doi: 10.1148/radiol.2492080169. [PubMed: 18936319].

6. Fanucci E, Fiaschetti V, Rotili A, Floris R, Simonetti G. Whole body 16-row multislice CT in emergency room: effects of different protocols on scanning time, image quality and radiation exposure. Emerg Radiol. 2007;13(5):251-7. doi: 10.1007/s10140-006-0554-0. [PubMed: 17180674].

7. Stokkeland PJ, Andersen E, Bjorndal MM, Mikalsen AM, Aslaksen S, Hyldmo PK. Maintaining immobilisation devices on trauma patients during CT: A feasibility study. Scand J Trauma Resusc Emerg Med. 2017;25(1):84. doi: 10.1186/s13049-017-0428-3. [PubMed: 28835284]. [PubMed Central: PMC5569509].

8. European Study Group of Radiologists and Physicists. European guidelines on quality criteria for computed tomography. Luxembourg: European Commission; 2000.

9. Macari M, Chandarana H, Schmidt B, Lee J, Lamparello P, Babb J. Abdominal aortic aneurysm: Can the arterial phase at CT evaluation after endovascular repair be eliminated to reduce radiation dose? Radiology. 2006;241(3):908-14. doi: 10.1148/radiol.2413051571. [PubMed: 17065562].

10. Karlo C, Gnannt R, Frauenfelder T, Leschka S, Bruesch M, Wanner GA, et al. Whole-body CT in polytrauma patients: Effect of arm positioning on thoracic and abdominal image quality. Emerg Radiol. 2011;18(4):285-93. doi: 10.1007/s10140-011-0948-5. [PubMed: 21472460].

11. Hoppe H, Vock P, Bonel HM, Ozdoba C, Gralla J. A novel multiple- trauma CT-scanning protocol using patient repositioning. Emerg Radiol. 2006;13(3):123-8. doi: 10.1007/s10140-006-0490-z. [PubMed: 17039342].

12. Bayer J, Pache G, Strohm PC, Zwingmann J, Blanke P, Baumann T, et al. Influence of arm positioning on radiation dose for whole body computed tomography in trauma patients. J Trauma. 2011;70(4):9005. doi: 10.1097/TA.0b013e3181edc80e. [PubMed: 20962679].

13. Huber-Wagner S, Lefering R, Qvick LM, Korner M, Kay MV, Pfeifer KJ, et al. Effect of whole-body CT during trauma resuscitation on survival: A retrospective, multicentre study. Lancet. 2009;373(9673):1455-61. doi: 10.1016/S0140-6736(09)60232-4. [PubMed:19321199].

14. Munk RD, Strohm PC, Saueressig U, Zwingmann J, Uhl M, Sudkamp $\mathrm{NP}$, et al. Effective dose estimation in whole-body multislice CT in paediatric trauma patients. Pediatr Radiol. 2009;39(3):245-52. doi: 10.1007/s00247-008-1091-7. [PubMed: 19148630].

15. Deunk J, Brink M, Dekker HM, Kool DR, van Kuijk C, Blickman JG, et al. Routine versus selective computed tomography of the abdomen, pelvis, and lumbar spine in blunt trauma: A prospective evaluation. J Trauma. 2009;66(4):1108-17. doi: 10.1097/TA.0b013e31817e55c3. [PubMed: 19359922].

16. Kortesniemi M, Kiljunen T, Kangasmaki A. Radiation exposure in body computed tomography examinations of trauma patients. Phys Med Biol. 2006;51(12):3269-82. doi: 10.1088/0031-9155/51/12/018. [PubMed: 16757876].

17. Rohrbein F, Goddard P, Schneider M, James G, Guo K. How does image noise affect actual and predicted human gaze allocation in assessing image quality? Vision Res. 2015;112:11-25. doi: 10.1016/j.visres.2015.03.029. [PubMed: 25982711].

18. McCollough $\mathrm{CH}$, Bruesewitz MR, Kofler JM Jr. CT dose reduction and dose management tools: Overview of available options. Radiographics. 2006;26(2):503-12. doi: 10.1148/rg.262055138. [PubMed: 16549613].

19. Nagel HD. Functional description of dose right 2.0. Philips healthcare. 2008. Available from: https://www.scribd.com/document/ 259301247/White-Paper-Dose-Right-2-0.

20. Soderberg M, Gunnarsson M. Automatic exposure control in computed tomography-an evaluation of systems from different manufacturers. Acta Radiol. 2010;51(6):625-34. doi: 10.3109/02841851003698206. [PubMed: 20429764]. 